\title{
Treatment Outcomes of HIV Infected Children After Initiation of Antiretroviral Therapy in Southwest China: an Observational Cohort Study
}

\section{Xiaoliang Zeng}

Guangxi Zhuang Autonomous Region Center for Disease Control and Prevention https://orcid.org/0000-00032201-3489

huanhuan chen

Guangxi Zhuang Autonomous Region Center for Disease Control and Prevention qiuying zhu

Guangxi Zhuang Autonomous Region Center for Disease Control and Prevention zhiyong shen

Guangxi Zhuang Autonomous Region Center for Disease Control and Prevention guanghua lan

Guangxi Zhuang Autonomous Region Center for Disease Control and Prevention jiangming liang

Guangxi Zhuang Autonomous Region Center for Disease Control and Prevention fuxiong liang

Guangxi Zhuang Autonomous Region Center for Disease Control and Prevention jinhui zhu

Guangxi Zhuang Autonomous Region Center for Disease Control and Prevention hui xing

Chinese Center for Disease Control and Prevention

\section{yiming shao}

Chinese Center for Disease Control and Prevention

\section{yuhua ruan}

Guangxi Zhuang Autonomous Region Center for Disease Control and Prevention

\section{jianfeng zhang}

Guangxi Medical University

\section{Xiangjun Zhang ( $\sim$ zxj@nevada.unr.edu )}

University of Tennessee Center for Public Health: The University of Tennessee Knoxville Department of Public Health

\section{Research Article}

Keywords: HIV, Children, Mortality, Attrition, Antiretroviral therapy

Posted Date: September 3rd, 2021 
DOI: https://doi.org/10.21203/rs.3.rs-829348/v1

License: (c) (i) This work is licensed under a Creative Commons Attribution 4.0 International License. Read Full License 


\section{Abstract}

Background: The number of HIV infected children receiving antiviral treatment in Guangxi is increasing. Understanding factors and trends of mortality and attrition in HIV-infected children under antiretroviral therapy (ART) was an urgently need to improve treatment outcomes. This study aimed to describe and assess mortality and attrition after ART initiation between 2004 and 2018 among children with HIV in Guangxi, China.

Methods: Cohort study data were extracted from the National Free Antiretroviral Treatment Program (NFATP) database, which has standard guidelines for core treatment indicators and other data at all HIV/AIDS treatment facilities of Guangxi. A total of $901 \mathrm{HIV}$-infected children who have started ART were included in the study. The study collected the following data: age, gender, WHO clinic stages before ART, CD4 cell count before ART, Cotrimoxazole prophylaxison (CTX) use before ART, initial ART regimen, malnutrition before ART, abnormal liver function before ART, abnormal kidney function before ART, severe anemia before ART, and time lag between a HIV diagnosis and ART initiation.

Results: HIV-infected children under ART had a mortality rate of 0.87 per 100 person-years, and an attrition rate of 3.02 per 100 person-years. Mortality was lower among children with CD 4 count between 200-500 copies/ml (Adjusted Hazard Ratio (AHR) 0.22, 95\% Confidence Interval (Cl) 0.09-0.54), and CD4 count $\geq 500 \mathrm{copies} / \mathrm{ml}$ (AHR 0.09, 95\% Cl 0.03-0.27); but higher among children with late ART initiation at 1-3 months (AHR 2.37, 95\% Cl 1.105.08), and at $\geq 3$ months (AHR 2.22, 95\% Cl 1.05-4.72). Attrition was lower among children with a CD4 count $\geq 500$ copies/ml (AHR 0.61, 95\% Cl 0.40-0.93), but higher among children with late ART initiation at 1-3 months (AHR 1.63, 95\% Cl 1.10-2.42), and at $\geq 3$ months (AHR 1.26, 95\% Cl 0.85-1.86).

Conclusion: Supportive programs are needed to educate children's families and parents on early ART, link HIVinfected children to care, and retain them in care among other programs that treat and manage physical conditions of HIV-infected children before ART initiation.

\section{Introduction}

Despite intensive efforts for the prevention of mother-to-child transmission (MTCT), HIV consistently impacts children. Globally 1.8 million children aged < 15 years living with HIV/AIDS, and approximately 150,000 children became newly infected and 95,000 children died of HIV/AIDS-related illnesses in 2019.(1) Children HIV disproportionally impacted middle- and low- income countries where HIV disease burden was high and resources were limited. $(2,3)$ China's MTCT prevention programs have achieved full coverage in 2015 , although significant improvement has been made, the MTCT rate was as high as 5.7 in $2016 \%(4,5)$ Moreover, the leading causes of death among infectious diseases in Chinese students aged 6 to 22 years shifted from rabies and tuberculosis in 2008 to HIV/AIDS in 2017.(6)

Without timely antiretroviral therapy (ART), the mortality of HIV-infected young children significantly increased (52.5\% vs $7.6 \%$ uninfected at the age of 2).(7) On the other hand, early HIV diagnosis and early ART initiation substantially reduced infant mortality.(8) ART was recommended by World Health Organization (WHO) for children infected with HIV regardless of age, CD4 status, and clinical stage as of 2015.(9) However, children ART coverage remained low worldwide, only approximately 53\% children aged $<15$ years have received ART compared to $67 \%$ of people aged 15 and older in 2019. $(10,11)$ A nationwide study reported that as of 2018 , only 5892 (73.4\%) out of 8029 HIV-infected children $\leq 14$ years have started ART in China.(12) Although ART had 
significantly extended children's survival time,(12) HIV-infected children had much lower rates on the receipt of ART compared to the national average ART coverage of $80.4 \%$ in China.(13) Furthermore, HIV-infected children might encounter challenges such as parental death, school dropout, and stigma which in turn resulted in negative consequences.(7)

Southern and southwestern China reported the highest HIV disease burden nationwide.(14) Guangxi Zhuang Autonomous Region that is located in southern China sharing borders with "Golden Triangle" ranked 3rd for HIV cases in 2018. $(14,15)$ It was one of six provinces and autonomous regions that had an HIV prevalence in pregnant women of over 50 per 100,000 population. $(14,16)$ MTCT programs have been scaled up in Guangxi and contributed to a significant decrease of MTCT in recent years.(17) Although early ART has been proven to be effective and beneficial for HIV-infected children, limited studies examined factors associated with death and attrition among HIV-infected children who were receiving ART. This retrospective study aimed to investigate factors that were related to death and attrition among a cohort of HIV-infected children who initiated ART. The findings of this study could be used to design programs with the goals to improve treatment outcomes and maximize the benefits of ART in HIV-infected children.

\section{Methods}

\section{Patient and public Involvement:}

Participants and the public were not involved in the design and administration of the study.

\section{Study design and participants}

The observational cohort study was conducted in Guangxi. Eligibility criteria included: (1). HIV positive children younger than 14 years old; (2). Enrolled in the National Free Antiretroviral Treatment Program (NFATP) between January 1, 2004 and December 31, 2018; (3). Being willing to provide informed consent for study participation. The study did not include participants who have not started ART. NFATP included approximately all HIV-infected children who were receiving ART in Guangxi. The study was approved by the institutional review board of Guangxi Center for Disease Control and Prevention. The research was performed in accordance with approved procedures.

\section{Data collection}

Cohort study data were extracted from the NFATP database. The following baseline data of study participants were collected: age, gender, clinic stage before ART (using WHO's criteria), CD4 count before ART, cotrimoxazole prophylaxison (CTX) use before ART, initial ART regimen, malnutrition status before ART, liver function before ART, kidney function before ART, anemia status before ART, and time between a HIV diagnosis and ART initiation. Follow-up data that were collected included: death, cessation of ART, and loss to follow-up. Date of death was based on death certificate information. Lost to follow-up was defined as missing more than 90 days after the date of last ART clinic visit, which was also defined as the date of ART cessation. More details could be found at a previously published article that used the Chinese national HIV treatment cohort study databases.(18)

China has been providing free ART for all HIV-infected individuals. The most common ART regimens that were prescribed to HIV-infected children were zidovudine (AZT), lamivudine (3TC), and nevirapine (NVP)/efavirenz (EFV)/lopinavir; or abacavir (ABC), lamivudine (3TC), and nevirapine (NVP)/efavirenz (EFV)/lopinavir. We 
categorized ATR regimens into the following three groups: containing AZT, containing ABC, and others.(19) Malnutrition was defined as BMI-for-age Z-score (BMIZ) less than - 3SD that was recommended by WHO for children and adolescents.(20) Abnormal liver function was defined as either alanine transaminase (ALT) or aspartate transaminase (AST) was higher than lab references. A cutoff point for AST and ALT level was set at 40 IU/L.(21) Abnormal kidney function was defined when serum creatinine (Scr) was higher than the lab reference. Scr thresholds using the enzymatic method were set up for different age groups: 0-7 days, $1.19 \mathrm{mg} / \mathrm{ml} ; 7$ days$1 \mathrm{month}, 0.79 \mathrm{mg} / \mathrm{ml} ; 1$ month-1 year, $0.50 \mathrm{mg} / \mathrm{ml} ; 1-10$ years, $1.09 \mathrm{mg} / \mathrm{ml}$; and $10-19$ years, $1.29 \mathrm{mg} / \mathrm{ml} .(22)$ Severe anemia was defined as a less than $8 \mathrm{~g} / \mathrm{dl}$ of hemoglobin concentration value.(23)

\section{Statistical analysis}

We performed a time-to-event cohort analysis. The primary study endpoints were death and attrition. Attrition was defined as lost to follow-up or cessation of ART as recorded in the NFATP database. Mortality and attrition rates were calculated based on Poisson distributions and their $95 \%$ confidence intervals (CI) were assessed with incidence densities per 100 person-years at follow-up. Data were censored on Dec 31, 2019.

Cox proportional hazard models were performed to evaluate treatment effect of initial ART regimens on death and attrition (cessation of ART or loss to follow-up) of HIV-infected children who started ART between 2004 and 2018, respectively. Competing risks for cause-specific hazard models were censored accordingly. A two-sided pvalue of $\leq 0.05$ was regarded as statistically significant. Statistical Analysis System (SAS $9.1^{\text {Tw }}$ for Windows; SAS Institute Inc., NC, USA) was used for all data analyses.

\section{Results}

\section{Baseline characteristics of study participants}

Of 911HIV-infected children who started ART from 2004 to 2018 in Guangxi, 10 participants were older than 14 years at ART initiation. A total of 901 study participants were included in the final cohort study analyses. Regarding participants' characteristics at ART initiation, 55.8\% of participants were less than 5 years old, more than half of participants were males (52.3\%), and more than one third were at a clinic stage of III or IV (35.1\%, Table 1). Prior to ART initiation, 38.0\% participants had a very low CD4 cell count of $<200 \mathrm{copies} / \mathrm{ml}$, one fourth had a CD 4 count of $200-500$ copies $/ \mathrm{ml}$, and $37 \%$ participants had a CD 4 count of $\geq 500$ copies $/ \mathrm{ml}$. Nearly half of HIV-infected children have used CTX before ART initiation (47.8\%). The initial ART regimens used by participants included ART containing AZT (71.6\%), ART containing ABC (20.5\%), and other (7.9\%). Furthermore, the proportions of HIV-infected children who met definitions of malnutrition, abnormal liver function, abnormal kidney function, and severe anemia were $6.0 \%, 43.6 \%, 14.9 \%$ and $4.1 \%$, respectively. More than one third of participants initiated ART within 1 month after receiving a HIV diagnosis (36.5\%). 
Table 1

Characteristics of HIV infected children started ART in Guangxi, 20042018

\begin{tabular}{|c|c|c|}
\hline Variable & Number & $\%$ \\
\hline Total & 901 & 100.0 \\
\hline \multicolumn{3}{|l|}{ Age before ART } \\
\hline $0-5$ years & 503 & 55.8 \\
\hline$\geq 5$ years & 398 & 44.2 \\
\hline \multicolumn{3}{|l|}{ Gender } \\
\hline Male & 471 & 52.3 \\
\hline Female & 430 & 47.7 \\
\hline \multicolumn{3}{|c|}{ WHO stage before ART } \\
\hline $1 / 2$ & 585 & 64.9 \\
\hline $3 / 4$ & 316 & 35.1 \\
\hline \multicolumn{3}{|l|}{ CD4 count before ART } \\
\hline <200 copies/ml & 342 & 38.0 \\
\hline 200-500 copies/ml & 225 & 25.0 \\
\hline$\geq 500$ copies $/ m l$ & 334 & 37.0 \\
\hline \multicolumn{3}{|l|}{ CTX use before ART } \\
\hline No & 431 & 47.8 \\
\hline Yes & 470 & 52.2 \\
\hline \multicolumn{3}{|l|}{ Initial ART regimen } \\
\hline Cotaining AZT & 645 & 71.6 \\
\hline Cotaining $A B C$ & 185 & 20.5 \\
\hline Others & 71 & 7.9 \\
\hline \multicolumn{3}{|c|}{ Malnutrition before ART } \\
\hline No & 847 & 94.0 \\
\hline Yes & 54 & 6.0 \\
\hline \multicolumn{3}{|c|}{ Abnormal liver function before ART } \\
\hline No & 508 & 56.4 \\
\hline Yes & 393 & 43.6 \\
\hline
\end{tabular}




\begin{tabular}{|lll|}
\hline Variable & Number & $\%$ \\
\hline No & 767 & 85.1 \\
\hline Yes & 134 & 14.9 \\
\hline Severe anemia before ART & & \\
\hline No & 864 & 95.9 \\
\hline Yes & 37 & 4.1 \\
\hline Time lag since HIV diagnosis and start of ART & & \\
\hline$<1$ month & 329 & 36.5 \\
\hline $1-3$ months & 244 & 27.1 \\
\hline$\geq 3$ months & 328 & 36.4 \\
\hline
\end{tabular}

\section{Mortality}

Among 901 HIV-infected children who started ART between 2004 and 2018, 47 of them died, the average mortality rate was 0.87 deaths per 100 person-years. The mortality rate per 100 person-years was 3.65 in the first year of ART, and it decreased thereafter (Table 2). Univariate Cox regression analyses indicated that several factors were significantly associated with mortality, including status before ART started such as disease severity (WHO clinic stage), CD4 count, malnutrition, and abnormal liver function, initial ART regimen, and time lag between a HIV diagnosis and ART initiation. In the multivariate model, higher levels of CD4 count were associated with reduced risks of mortality (200-500, AHR 0.22, CI 0.09-0.55; $\geq 500$, AHR 0.10, CI 0.03-0.29, Table 3). Furthermore, longer time lag between a HIV diagnosis and ART initiation was associated with increased risks of mortality (1-3 months, AHR 2.30, CI 1.07-4.94; $\geq 3$ months, AHR 2.22, CI 1.04-4.74). 
Table 2

Mortality and attrition rates of HIV infected children started ART in Guangxi, 2004-2018

\begin{tabular}{|c|c|c|c|c|c|c|}
\hline Variable & Number & Deaths & $\begin{array}{l}\text { Person } \\
\text { years }\end{array}$ & $\begin{array}{l}\text { Deaths per } 100 \\
\text { person years }(95 \% \mathrm{Cl})\end{array}$ & Attritions & $\begin{array}{l}\text { Attritions per } 100 \\
\text { person years }(95 \% \\
\text { Cl) }\end{array}$ \\
\hline Total & 901 & 47 & 5394.48 & $0.87(0.63-1.11)$ & 163 & $3.02(2.57-3.47)$ \\
\hline \multicolumn{7}{|l|}{$\begin{array}{l}\text { Year post-ART } \\
\text { initiation }\end{array}$} \\
\hline The first year & 901 & 31 & 849.66 & $3.65(2.40-4.90)$ & 44 & $5.18(3.69-6.67)$ \\
\hline $\begin{array}{l}\text { The second } \\
\text { year }\end{array}$ & 839 & 2 & 825.08 & $0.24(0.00-0.57)$ & 24 & $2.91(1.77-4.04)$ \\
\hline The third year & 796 & 5 & 780.41 & $0.64(0.09-1.19)$ & 20 & $2.56(1.47-3.66)$ \\
\hline $\begin{array}{l}\text { The fourth } \\
\text { year }\end{array}$ & 722 & 2 & 705.41 & $0.28(0.00-0.67)$ & 23 & $3.26(1.96-4.56)$ \\
\hline The 5th year & 663 & 2 & 652.58 & $0.31(0.00-0.72)$ & 19 & $2.91(1.64-4.19)$ \\
\hline The 6th year & 577 & 3 & 566.22 & $0.53(0.00-1.11)$ & 14 & $2.47(1.21-3.74)$ \\
\hline The 7th year & 502 & 1 & 488.53 & $0.20(0.00-0.60)$ & 10 & $2.05(0.81-3.28)$ \\
\hline The 8th year & 394 & 0 & 389.23 & - & 2 & $0.51(0.00-1.21)$ \\
\hline The 9th year & 321 & 0 & 316.96 & - & 2 & $0.63(0.00-1.48)$ \\
\hline The 10th year & 255 & 1 & 247.42 & $0.40(0.00-1.18)$ & 2 & $0.81(0.00-1.90)$ \\
\hline The 11th year & 177 & 0 & 167.39 & - & 2 & $1.19(0.00-2.81)$ \\
\hline The 12th year & 113 & 0 & 109.48 & - & 0 & - \\
\hline The 13th year & 50 & 0 & 47.32 & - & 1 & $2.11(0.00-6.15)$ \\
\hline The 14th year & 24 & 0 & 22.88 & - & 0 & - \\
\hline The 15th year & 9 & 0 & 8.55 & - & 0 & - \\
\hline
\end{tabular}


Table 3

Mortality rates and risk factors of HIV infected children started ART in Guangxi, 2004-2018

\begin{tabular}{|c|c|c|c|c|c|c|c|c|}
\hline Variable & Number & Deaths & $\begin{array}{l}\text { Person- } \\
\text { years }\end{array}$ & $\begin{array}{l}\text { Deaths per } \\
100 \\
\text { person- } \\
\text { years ( } 95 \% \\
\text { Cl) }\end{array}$ & $\begin{array}{l}\text { HR } \\
(95 \% \mathrm{Cl})\end{array}$ & $p$ & $\begin{array}{l}\text { AHR } \\
(95 \% \mathrm{Cl})\end{array}$ & $P$ \\
\hline Total & 901 & 47 & 5394.48 & $\begin{array}{l}0.87(0.63- \\
1.11)\end{array}$ & & & & \\
\hline \multicolumn{9}{|l|}{$\begin{array}{l}\text { Age before } \\
\text { ART }\end{array}$} \\
\hline $0-5$ years & 503 & 25 & 3525.65 & $\begin{array}{l}0.71(0.44- \\
0.98)\end{array}$ & Reference & & Reference & \\
\hline$\geq 5$ years & 398 & 22 & 1868.83 & $\begin{array}{l}1.18(0.70- \\
1.66)\end{array}$ & $\begin{array}{l}1.24(0.69- \\
2.20)\end{array}$ & 0.473 & $\begin{array}{l}0.71(0.38- \\
1.34)\end{array}$ & 0.293 \\
\hline \multicolumn{9}{|l|}{ Gender } \\
\hline Male & 471 & 21 & 2948.97 & $\begin{array}{l}0.71(0.42- \\
1.01)\end{array}$ & Reference & & Reference & \\
\hline Female & 430 & 26 & 2445.51 & $\begin{array}{l}1.06(0.66- \\
1.46)\end{array}$ & $\begin{array}{l}1.39(0.78- \\
2.46)\end{array}$ & 0.267 & $\begin{array}{l}1.77(0.97- \\
3.23)\end{array}$ & 0.064 \\
\hline \multicolumn{9}{|l|}{$\begin{array}{l}\text { WHO stage } \\
\text { before ART }\end{array}$} \\
\hline $1 / 2$ & 585 & 20 & 3457.10 & $\begin{array}{l}0.58(0.33- \\
0.83)\end{array}$ & Reference & & Reference & \\
\hline $3 / 4$ & 316 & 27 & 1937.39 & $\begin{array}{l}1.39(0.88- \\
1.91)\end{array}$ & $\begin{array}{l}2.56(1.44- \\
4.57)\end{array}$ & 0.001 & $\begin{array}{l}1.44(0.75- \\
2.75)\end{array}$ & 0.269 \\
\hline \multicolumn{9}{|l|}{$\begin{array}{l}\text { CD4 count } \\
\text { before ART }\end{array}$} \\
\hline $\begin{array}{l}<200 \\
\text { copies/ml }\end{array}$ & 342 & 37 & 1857.02 & $\begin{array}{l}1.99(1.37- \\
2.62)\end{array}$ & Reference & & Reference & \\
\hline $\begin{array}{l}200-500 \\
\text { copies/ml }\end{array}$ & 225 & 6 & 1430.9 & $\begin{array}{l}0.42(0.09- \\
0.75)\end{array}$ & $\begin{array}{l}0.23(0.1- \\
0.54)\end{array}$ & 0.001 & $\begin{array}{l}0.22(0.09- \\
0.55)\end{array}$ & 0.001 \\
\hline $\begin{array}{l}\geq 500 \\
\text { copies/ml }\end{array}$ & 334 & 4 & 2106.56 & $\begin{array}{l}0.19(0.01- \\
0.37)\end{array}$ & $\begin{array}{l}0.10(0.04- \\
0.29)\end{array}$ & $\begin{array}{l}< \\
0.001\end{array}$ & $\begin{array}{l}0.10(0.03- \\
0.29)\end{array}$ & $\begin{array}{l}< \\
0.001\end{array}$ \\
\hline \multicolumn{9}{|l|}{$\begin{array}{l}\text { CTX use } \\
\text { before ART }\end{array}$} \\
\hline No & 431 & 19 & 2107.14 & $\begin{array}{l}0.90(0.51- \\
1.30)\end{array}$ & Reference & & Reference & \\
\hline Yes & 470 & 28 & 3287.34 & $\begin{array}{l}0.85(0.54- \\
1.16)\end{array}$ & $\begin{array}{l}1.23(0.68- \\
2.20)\end{array}$ & 0.495 & $\begin{array}{l}0.60(0.31- \\
1.17)\end{array}$ & 0.132 \\
\hline
\end{tabular}

HR: hazard ratio in univariate regression; AHR: adjusted hazard ratio in multivariate regression. 


\begin{tabular}{|c|c|c|c|c|c|c|c|c|}
\hline Variable & Number & Deaths & $\begin{array}{l}\text { Person- } \\
\text { years }\end{array}$ & $\begin{array}{l}\text { Deaths per } \\
100 \\
\text { person- } \\
\text { years }(95 \% \\
\text { Cl) }\end{array}$ & $\begin{array}{l}\text { HR } \\
(95 \% \mathrm{Cl})\end{array}$ & $p$ & $\begin{array}{l}\text { AHR } \\
(95 \% \mathrm{Cl})\end{array}$ & $P$ \\
\hline \multicolumn{9}{|l|}{$\begin{array}{l}\text { Initial ART } \\
\text { regimen }\end{array}$} \\
\hline $\begin{array}{l}\text { Cotaining } \\
\text { AZT }\end{array}$ & 645 & 27 & 4104.26 & $\begin{array}{l}0.66(0.42- \\
0.90)\end{array}$ & Reference & & Reference & \\
\hline $\begin{array}{l}\text { Cotaining } \\
\text { ABC }\end{array}$ & 185 & 12 & 836.28 & $\begin{array}{l}1.43(0.64- \\
2.23)\end{array}$ & $\begin{array}{l}1.69(0.85- \\
3.34)\end{array}$ & 0.132 & $\begin{array}{l}1.17(0.54- \\
2.55)\end{array}$ & 0.693 \\
\hline Others & 71 & 8 & 453.94 & $\begin{array}{l}1.76(0.57- \\
2.95)\end{array}$ & $\begin{array}{l}2.77(1.26- \\
6.09)\end{array}$ & 0.012 & $\begin{array}{l}1.49(0.62- \\
3.57)\end{array}$ & 0.374 \\
\hline \multicolumn{9}{|c|}{$\begin{array}{l}\text { Malnutrition } \\
\text { before ART }\end{array}$} \\
\hline No & 847 & 41 & 5104.40 & $\begin{array}{l}0.80(0.56- \\
1.04)\end{array}$ & Reference & & Reference & \\
\hline Yes & 54 & 6 & 290.08 & $\begin{array}{l}2.07(0.45- \\
3.68)\end{array}$ & $\begin{array}{l}2.45(1.04- \\
5.76)\end{array}$ & 0.041 & $\begin{array}{l}1.84(0.74- \\
4.56)\end{array}$ & 0.188 \\
\hline \multicolumn{9}{|c|}{$\begin{array}{l}\text { Abnormal } \\
\text { liver function } \\
\text { before ART }\end{array}$} \\
\hline No & 508 & 20 & 2826.21 & $\begin{array}{l}0.71(0.41- \\
1.01)\end{array}$ & Reference & & Reference & \\
\hline Yes & 393 & 27 & 2568.27 & $\begin{array}{l}1.05(0.66- \\
1.44)\end{array}$ & $\begin{array}{l}1.71(0.96- \\
3.05)\end{array}$ & 0.07 & $\begin{array}{l}1.36(0.73- \\
2.51)\end{array}$ & 0.331 \\
\hline \multicolumn{9}{|c|}{$\begin{array}{l}\text { Abnormal } \\
\text { kidney } \\
\text { function } \\
\text { before ART }\end{array}$} \\
\hline No & 767 & 37 & 4316.97 & $\begin{array}{l}0.86(0.59- \\
1.13)\end{array}$ & Reference & & Reference & \\
\hline Yes & 134 & 10 & 1077.51 & $\begin{array}{l}0.93(0.37- \\
1.49)\end{array}$ & $\begin{array}{l}1.41(0.70- \\
2.86)\end{array}$ & 0.334 & $\begin{array}{l}1.23(0.54- \\
2.80)\end{array}$ & 0.615 \\
\hline \multicolumn{9}{|c|}{$\begin{array}{l}\text { Severe } \\
\text { anemia } \\
\text { before ART }\end{array}$} \\
\hline No & 864 & 44 & 5122.72 & $\begin{array}{l}0.86(0.61- \\
1.11)\end{array}$ & Reference & & Reference & \\
\hline Yes & 37 & 3 & 271.76 & $\begin{array}{l}1.10(0.00- \\
2.32)\end{array}$ & $\begin{array}{l}1.47(0.46- \\
4.75)\end{array}$ & 0.516 & $\begin{array}{l}1.46(0.41- \\
5.16)\end{array}$ & 0.557 \\
\hline
\end{tabular}

HR: hazard ratio in univariate regression; AHR: adjusted hazard ratio in multivariate regression. 


\begin{tabular}{|c|c|c|c|c|c|c|c|c|}
\hline Variable & Number & Deaths & $\begin{array}{l}\text { Person- } \\
\text { years }\end{array}$ & $\begin{array}{l}\text { Deaths per } \\
100 \\
\text { person- } \\
\text { years }(95 \% \\
\text { Cl) }\end{array}$ & $\begin{array}{l}\mathrm{HR} \\
(95 \% \mathrm{Cl})\end{array}$ & p & $\begin{array}{l}\text { AHR } \\
(95 \% \mathrm{Cl})\end{array}$ & P \\
\hline \multicolumn{9}{|l|}{$\begin{array}{l}\text { Time lag } \\
\text { since HIV } \\
\text { diagnosis } \\
\text { and start of } \\
\text { ART }\end{array}$} \\
\hline$<1$ month & 329 & 11 & 2091.88 & $\begin{array}{l}0.53(0.22- \\
0.83)\end{array}$ & Reference & & Reference & \\
\hline $1-3$ months & 244 & 17 & 1433.91 & $\begin{array}{l}1.19(0.64- \\
1.74)\end{array}$ & $\begin{array}{l}2.14(1.00- \\
4.58)\end{array}$ & 0.049 & $\begin{array}{l}2.30(1.07- \\
4.94)\end{array}$ & 0.033 \\
\hline$\geq 3$ months & 328 & 19 & 1868.69 & $\begin{array}{l}1.02(0.57- \\
1.46)\end{array}$ & $\begin{array}{l}1.77(0.84- \\
3.71)\end{array}$ & 0.134 & $\begin{array}{l}2.22(1.04- \\
4.74)\end{array}$ & 0.04 \\
\hline
\end{tabular}

\section{Attrition}

Among 901 HIV-infected children who started ART between 2004 and 2018, 163 attritions were reported, and the average attrition rate was 3.02 deaths per 100 person-years. The attrition rate per 100 person-years was 5.18 in the first year of ART, and decreased after then (Table 2). Using univariate Cox regression analyses, factors that were significantly associated with attrition were determined: age before ART, disease severity (WHO clinic stage) before ART, CD4 count before ART, initial ART regimen, and time lag between a HIV diagnosis and ART initiation. In the multivariate model, a high CD4 level was associated with a reduced risk of attrition rate ( $\geq 500, A H R 0.62$; Cl 0.41-0.91, Table 4). On the other hand, a delayed initiation of ART ( $\geq 3$ months) was associated with an increased risk of attrition (AHR 1.55, Cl 1.05-2.30). 
Table 4

Attrition rates and risk factors of HIV infected children started ART in Guangxi, 2004-2018

\begin{tabular}{|c|c|c|c|c|c|c|c|c|}
\hline Variable & Number & Attritions & $\begin{array}{l}\text { Person- } \\
\text { years }\end{array}$ & $\begin{array}{l}\text { Attritions } \\
\text { per } \\
100 \\
\text { person- } \\
\text { years ( } 95 \% \\
\text { Cl) }\end{array}$ & $\begin{array}{l}\text { HR } \\
(95 \% \mathrm{Cl})\end{array}$ & $p$ & $\begin{array}{l}\text { AHR } \\
(95 \% \mathrm{Cl})\end{array}$ & $\mathbf{P}$ \\
\hline Total & 901 & 163 & 5394.48 & $\begin{array}{l}3.02(2.57- \\
3.47)\end{array}$ & & & & \\
\hline \multicolumn{9}{|l|}{$\begin{array}{l}\text { Age before } \\
\text { ART }\end{array}$} \\
\hline $0-5$ years & 503 & 84 & 3525.65 & $\begin{array}{l}2.38(1.89- \\
2.88)\end{array}$ & Reference & & Reference & \\
\hline$\geq 5$ years & 398 & 79 & 1868.83 & $\begin{array}{l}4.23(3.32- \\
5.14)\end{array}$ & $\begin{array}{l}1.56(1.14- \\
2.13)\end{array}$ & 0.005 & $\begin{array}{l}1.32(0.92- \\
1.89)\end{array}$ & 0.131 \\
\hline \multicolumn{9}{|l|}{ Gender } \\
\hline Male & 471 & 76 & 2948.97 & $\begin{array}{l}2.58(2.01- \\
3.14)\end{array}$ & Reference & & Reference & \\
\hline Female & 430 & 87 & 2445.51 & $\begin{array}{l}3.56(2.83- \\
4.29)\end{array}$ & $\begin{array}{l}1.33(0.98- \\
1.81)\end{array}$ & 0.069 & $\begin{array}{l}1.39(1.01- \\
1.91)\end{array}$ & 0.041 \\
\hline \multicolumn{9}{|l|}{$\begin{array}{l}\text { WHO stage } \\
\text { before ART }\end{array}$} \\
\hline $1 / 2$ & 585 & 94 & 3457.1 & $\begin{array}{l}2.72(2.18- \\
3.25)\end{array}$ & Reference & & Reference & \\
\hline $3 / 4$ & 316 & 69 & 1937.39 & $\begin{array}{l}3.56(2.74- \\
4.38)\end{array}$ & $\begin{array}{l}1.37(1.01- \\
1.88)\end{array}$ & 0.046 & $\begin{array}{l}1.22(0.86- \\
1.73)\end{array}$ & 0.277 \\
\hline \multicolumn{9}{|l|}{$\begin{array}{l}\text { CD4 count } \\
\text { before ART }\end{array}$} \\
\hline $\begin{array}{l}<200 \\
\text { copies/ml }\end{array}$ & 342 & 77 & 1857.02 & $\begin{array}{l}4.15(3.24- \\
5.05)\end{array}$ & Reference & & Reference & \\
\hline $\begin{array}{l}200-500 \\
\text { copies/ml }\end{array}$ & 225 & 38 & 1430.9 & $\begin{array}{l}2.66(1.83- \\
3.48)\end{array}$ & $\begin{array}{l}0.65(0.44- \\
0.96)\end{array}$ & 0.030 & $\begin{array}{l}0.66(0.44- \\
1.01)\end{array}$ & 0.053 \\
\hline $\begin{array}{l}\geq 500 \\
\text { copies/ml }\end{array}$ & 334 & 48 & 2106.56 & $\begin{array}{l}2.28(1.65- \\
2.91)\end{array}$ & $\begin{array}{l}0.56(0.39- \\
0.81)\end{array}$ & 0.002 & $\begin{array}{l}0.62(0.41- \\
0.95)\end{array}$ & 0.026 \\
\hline
\end{tabular}

HR: hazard ratio in univariate regression; AHR: adjusted hazard ratio in multivariate regression. 


\begin{tabular}{|c|c|c|c|c|c|c|c|c|}
\hline Variable & Number & Attritions & $\begin{array}{l}\text { Person- } \\
\text { years }\end{array}$ & $\begin{array}{l}\text { Attritions } \\
\text { per } \\
100 \\
\text { person- } \\
\text { years (95\% } \\
\text { Cl) }\end{array}$ & $\begin{array}{l}\mathrm{HR} \\
(95 \% \mathrm{Cl})\end{array}$ & p & $\begin{array}{l}\text { AHR } \\
(95 \% \mathrm{Cl})\end{array}$ & $P$ \\
\hline \multicolumn{9}{|l|}{$\begin{array}{l}\text { CTX use } \\
\text { before ART }\end{array}$} \\
\hline No & 431 & 70 & 2107.14 & $\begin{array}{l}3.32(2.56- \\
4.08)\end{array}$ & Reference & & Reference & \\
\hline Yes & 470 & 93 & 3287.34 & $\begin{array}{l}2.83(2.27- \\
3.39)\end{array}$ & $\begin{array}{l}0.96(0.70- \\
1.31)\end{array}$ & 0.793 & $\begin{array}{l}0.87(0.60- \\
1.24)\end{array}$ & 0.430 \\
\hline \multicolumn{9}{|l|}{$\begin{array}{l}\text { Initial ART } \\
\text { regimen }\end{array}$} \\
\hline $\begin{array}{l}\text { Cotaining } \\
\text { AZT }\end{array}$ & 645 & 115 & 4104.26 & $\begin{array}{l}2.80(2.30- \\
3.30)\end{array}$ & Reference & & Reference & \\
\hline $\begin{array}{l}\text { Cotaining } \\
\text { ABC }\end{array}$ & 185 & 24 & 836.28 & $\begin{array}{l}2.87(1.75- \\
3.99)\end{array}$ & $\begin{array}{l}0.89(0.57- \\
1.38)\end{array}$ & 0.602 & $\begin{array}{l}0.75(0.47- \\
1.20)\end{array}$ & 0.231 \\
\hline Others & 71 & 24 & 453.94 & $\begin{array}{l}5.29(3.22- \\
7.35)\end{array}$ & $\begin{array}{l}1.97(1.27- \\
3.06)\end{array}$ & 0.003 & $\begin{array}{l}1.72(1.06- \\
2.78)\end{array}$ & 0.027 \\
\hline \multicolumn{9}{|c|}{$\begin{array}{l}\text { Malnutrition } \\
\text { before ART }\end{array}$} \\
\hline No & 847 & 155 & 5104.40 & $\begin{array}{l}3.04(2.57- \\
3.50)\end{array}$ & Reference & & Reference & \\
\hline Yes & 54 & 8 & 290.08 & $\begin{array}{l}2.76(0.89- \\
4.62)\end{array}$ & $\begin{array}{l}0.92(0.45- \\
1.86)\end{array}$ & 0.808 & $\begin{array}{l}0.79(0.38- \\
1.64)\end{array}$ & 0.53 \\
\hline \multicolumn{9}{|l|}{$\begin{array}{l}\text { Abnormal } \\
\text { liver } \\
\text { function } \\
\text { before ART }\end{array}$} \\
\hline No & 508 & 85 & 2826.21 & $\begin{array}{l}3.01(2.38- \\
3.63)\end{array}$ & Reference & & Reference & \\
\hline Yes & 393 & 78 & 2568.27 & $\begin{array}{l}3.04(2.38- \\
3.69)\end{array}$ & $\begin{array}{l}1.08(0.79- \\
1.47)\end{array}$ & 0.619 & $\begin{array}{l}1.07(0.77- \\
1.49)\end{array}$ & 0.686 \\
\hline \multicolumn{9}{|l|}{$\begin{array}{l}\text { Abnormal } \\
\text { kidney } \\
\text { function } \\
\text { before ART }\end{array}$} \\
\hline No & 767 & 134 & 4316.97 & $\begin{array}{l}3.10(2.59- \\
3.62)\end{array}$ & Reference & & Reference & \\
\hline Yes & 134 & 29 & 1077.51 & $\begin{array}{l}2.69(1.74- \\
3.65)\end{array}$ & $\begin{array}{l}0.99(0.66- \\
1.49)\end{array}$ & 0.968 & $\begin{array}{l}1.00(0.63- \\
1.59)\end{array}$ & 0.996 \\
\hline
\end{tabular}

HR: hazard ratio in univariate regression; AHR: adjusted hazard ratio in multivariate regression. 


\begin{tabular}{|c|c|c|c|c|c|c|c|c|}
\hline Variable & Number & Attritions & $\begin{array}{l}\text { Person- } \\
\text { years }\end{array}$ & $\begin{array}{l}\text { Attritions } \\
\text { per } \\
100 \\
\text { person- } \\
\text { years (95\% } \\
\text { Cl) }\end{array}$ & $\begin{array}{l}\text { HR } \\
(95 \% \mathrm{Cl})\end{array}$ & p & $\begin{array}{l}\text { AHR } \\
(95 \% \mathrm{Cl})\end{array}$ & P \\
\hline \multicolumn{9}{|l|}{$\begin{array}{l}\text { Severe } \\
\text { anemia } \\
\text { before ART }\end{array}$} \\
\hline No & 864 & 159 & 5122.72 & $\begin{array}{l}3.10(2.63- \\
3.57)\end{array}$ & Reference & & Reference & \\
\hline Yes & 37 & 4 & 271.76 & $\begin{array}{l}1.47(0.07- \\
2.88)\end{array}$ & $\begin{array}{l}0.50(0.19- \\
1.36)\end{array}$ & 0.176 & $\begin{array}{l}0.53(0.19- \\
1.48)\end{array}$ & 0.226 \\
\hline \multicolumn{9}{|l|}{$\begin{array}{l}\text { Time lag } \\
\text { since HIV } \\
\text { diagnosis } \\
\text { and start of } \\
\text { ART }\end{array}$} \\
\hline$<1$ month & 329 & 49 & 2091.88 & $\begin{array}{l}2.34(1.70- \\
2.98)\end{array}$ & Reference & & Reference & \\
\hline $\begin{array}{l}1-3 \\
\text { months }\end{array}$ & 244 & 53 & 1433.91 & $\begin{array}{l}3.70(2.73- \\
4.67)\end{array}$ & $\begin{array}{l}1.54(1.04- \\
2.27)\end{array}$ & 0.030 & $\begin{array}{l}1.55(1.05- \\
2.30)\end{array}$ & 0.029 \\
\hline$\geq 3$ months & 328 & 61 & 1868.69 & $\begin{array}{l}3.26(2.47- \\
4.06)\end{array}$ & $\begin{array}{l}1.30(0.89- \\
1.90)\end{array}$ & 0.169 & $\begin{array}{l}1.19(0.81- \\
1.77)\end{array}$ & 0.378 \\
\hline
\end{tabular}

\section{Discussion}

Globally, $14 \%$ of HIV-related deaths were children under the age of 15 although they only accounted for $5 \%$ of all people living with HIV/AIDS.(10) It is urgently needed to improve treatment outcomes for children with HIV. The study reported a mortality rate of 0.87 per 100 person-years in Guangxi HIV-infected children who have started ART. This result was lower than comparable reports from other Asian regions (2.1-2.86 per 100 person-years) for HIV-infected children. $(24,25)$ Also this mortality rate was much lower than HIV-infected adults who were receiving ART in Guangxi $(5.94$ per 100 person-years). $(26,27)$ Moreover, mortality reached the peak of 3.65 per 100 person-years within the first year, and dramatically reduced from the 2 nd year, and reached nearly 0 at the 8th year of ART initiation. The trends were consistent with previous studies that reported generally higher mortalities in the first 3-6 months of ART initiation and decreasing over time. $(24,28)$ It could be explained that infections and other complications related to advanced HIV disease and late clinic stages were the possible major causes of the first-year deaths because medication-related deaths were generally low.(28)

ART has significantly reduced HIV-infected children's mortality in this sample and initiating ART at a high CD4 level of $\geq 500$ contributed to the lowest mortality. On the other hand, low CD4 count and late ART initiation resulted in an increased mortality. In addition to a weakened immune system and its consequences, previous studies reported that a higher CD 4 count could reduce the odds of virologic failure and HIV drug resistance mutations.(29) However, 63.5\% participants have started ART after one month of a HIV diagnosis, and 38\% 
started ART with a very low CD4 count (<200 copies/ml) in this sample. And numbers of children have other physical conditions when they started ART, such as malnutrition, abnormal liver function, abnormal kidney function, and severe anemia. It suggested that supportive programs, such as nutritional support, routine examination, lab test are needed in addition to ART promotion.

The overall attrition rate was 3.02 per 100 person-years in this study (18.1\%). The result was consistent with the reports of Thailand (2.9 per 100 person-years) and Zambia (16\%) for HIV-infected children, $(25,30)$ but much lower than HIV-infected adults who started ART in Guangxi (10.86 per 100 person-years).(26) More than 25\% attritions occurred in the first year of ART initiation. High attrition rates can result in suboptimal treatment outcomes, increased mortality, increased cost of care, and excess infections.(31) Therefore, future interventions can provide retention support, such as case management, and follow-ups to help retain HIV-infected children in care. Moreover, health education and linkage to care services are needed to educate families with HIV-infected children to start ART as early as possible and remove possible barriers for ART initiation.

One strength of the study was it utilized a database which included approximately all HIV-infected children who were under ART in Guangxi. The study provided a profile of this group of children and assessed factors associated with mortality and attrition. The study also confirmed the effectiveness of pediatric ART. Therefore, the findings have important real-world implications to reduce mortality and attrition in HIV-infected children. The study has limitations. First, the study included HIV-infected children who were in NFATP between 2004 and 2018 in Guangxi, some groups were not covered such as, HIV-infected children who were not in ART. Therefore, the results cannot be generalized to these groups. Second, this sample and the number of events were relatively small (47 deaths), it possibly could cause over fitting of the Cox model to predict risk factors for mortality. Third, the retrospective study was limited by the existing information of the database. Some information that might be helpful to explain the study results was lacking, such as children's viral load, parental HIV status, and parental or families' attitudes or sociodemographic characteristics.

\section{Conclusion}

MTCT prevention efforts and ART have significantly reduced HIV-infected children's mortality although first-year mortality and attrition were relatively high after ART initiation. Low CD4 count and late ART initiation ( $>1$ month) were associated with increased mortality and attrition. The findings have implications to design interventions and programs that support linkage to care, early ART initiation, and first-year retention in care.

\section{Abbreviations}

AHR, adjusted hazard ratio; HR, hazard ratio.

\section{Declarations}

\section{Ethics approval and consent to participate}

The study was approved by the institutional review board of Guangxi Center for Disease Control and Prevention. The research was performed in accordance with approved procedures.

\section{Consent for publication}

Page 15/18 
All authors read and approved the final version of the paper.

\section{Availability of data and materials}

Data was extracted from the National Free Antiretroviral Treatment Program database. According to Chinese law, the original data can not be made public.

\section{Conflicts of interest}

The authors have no conflicts of interest to declare.

\section{Acknowledgments}

Data in this manuscript were collected by the Guangxi Center for Disease Control and Prevention. Principal investigators for this study included Q. Z., G. L., Z. S., J. L., F. L.(Guangxi Center for Disease Control and Prevention).

\section{Funding}

This work was supported by National Natural Science Foundation of China [11971479], Ministry of Science and Technology of China [2018ZX10721102-006, 2018ZX10715008], Guangxi Key Laboratory of AIDS Prevention Control and Translation [gklapt201903], Guangxi Bagui Honor Scholarship, and Chinese State Key Laboratory of Infectious Disease Prevention and Control.

\section{Role of the funding source}

The funders of the study had no role in study design, data collection, data analysis, data interpretation, or writing of the paper. The corresponding author has full access to all data in the study and takes final responsibility for the decision to submit for publication.

\section{Author contribution}

$X Z$, JZ and YR were responsible for study design and planning. QZ, ZS, GL, HC contributed to data collection and management. $X Z$, JZ, YR contributed to data analysis. $X Z, J Z, H Z, Y S, X Z$ and $Y R$ contributed to interpretation. $X Z$, $J Z, X Z, Y R$ contributed to writing the report.

\section{References}

1. UNAIDS. UNAIDS data 20202020 [5/13/2021]. Available from: https://www.unaids.org/sites/default/files/media_asset/2020_aids-data-book_en.pdf.

2. Iyun V, Technau K-G, Vinikoor M, Yotebieng M, Vreeman R, Abuogi L, et al. Variations in the characteristics and outcomes of children living with HIV following universal ART in sub-Saharan Africa (2006-17): a retrospective cohort study. 2021.

3. Rujumba J, Ndeezi GJTIH. Considerations for strengthening ART scale-up for children. 2021.

4. Dong Y, Guo W, Gui X, Liu Y, Yan Y, Feng L, et al. Preventing mother to child transmission of HIV: lessons learned from China. 2020;20(1):1-10. 
5. China National Working Committee on Children and Women under State Council. National mother-to-child transmission rate of HIV fell to $5.7 \%$ in 2016. 2017 [5/13/2021]. Available from:

http://www.nwccw.gov.cn/2017-12/04/content_187271.htm.

6. Dong Y, Wang L, Burgner DP, Miller JE, Song Y, Ren X, et al. Infectious diseases in children and adolescents in China: analysis of national surveillance data from 2008 to 2017. 2020;369.

7. Newell M-L, Coovadia H, Cortina-Borja M, Rollins N, Gaillard P, Dabis FJTL. Mortality of infected and uninfected infants born to HIV-infected mothers in Africa: a pooled analysis. 2004;364(9441):1236-43.

8. Violari A, Cotton MF, Gibb DM, Babiker AG, Steyn J, Madhi SA, et al. Early antiretroviral therapy and mortality among HIV-infected infants. 2008;359(21):2233-44.

9. World Health Organization. Consolidated guidelines on the use of antiretroviral drugs for treating and preventing HIV infection Recommendations for a public health approach - Second edition 2016 [5/13/2021]. Available from: https://www.who.int/hiv/pub/arv/arv-2016/en/.

10. UNICEF. Paediatric care and treatment 2020 [5/15/2021]. Available from: https://data.unicef.org/topic/hivaids/paediatric-treatment-and-care/.

11. UNAIDS. Global HIV \& AIDS statistics-2020 fact sheet 2021 [2/14/2021]. Available from: https://www.unaids.org/en/resources/fact-sheet.

12. Yin H, Ma Y, Yang X, Zhao H, Han MJZIxbxzzZIz. Survival analysis on HIV-infected children aged 14 years old and younger in China. 2020;41(6):850-5.

13. UNAIDS. Country progress report-China 2018 [5/13/2021]. Available from: https://www.unaids.org/sites/default/files/country/documents/CHN_2018_countryreport.pdf.

14. Wu Z, Chen J, Scott SR, McGoogan JMJCHAR. History of the HIV Epidemic in China. 2019;16(6):458-66.

15. Cao B, Saha PT, Leuba SI, Lu H, Tang W, Wu D, et al. Recalling, sharing and participating in a social media intervention promoting HIV testing: a longitudinal analysis of HIV testing among MSM in China. 2019;23(5):1240-9.

16. Jiang J, Zhou Y, Li H, Gao Y, Zhang Y, Luo S, et al. HIV epidemic among pregnant women in China, 2016: trend and spatial analysis. 2018;98(41):3360-4.

17. Zhao J, Chen Q, Fu C, Qin Q, Huang H, Feng Y, et al. Rate of the HIV Transmission and Associated Factors Among HIV-Exposed Infants in Guangxi, China: 2014-2019. 2020;36(8):647-55.

18. Huang X, Xu L, Sun L, Gao G, Cai W, Liu Y, et al. Six-year immunologic recovery and virological suppression of HIV patients on LPV/r-based second-line antiretroviral treatment: a multi-center real-world cohort study in China. 2019;10:1455.

19. Guo P-L, He H-L, Chen X-J, Chen J-F, Chen X-T, Lan Y, et al. Antiretroviral Long-Term Efficacy and Resistance of Lopinavir/Ritonavir Plus Lamivudine in HIV-1-Infected Treatment-Naïve Patients (ALTERLL): 144-Week Results of a Randomized, Open-Label, Non-Inferiority Study From Guangdong, China. 2020;11.

20. Kigaru DMD, Ndung'u ZW, Macharia-Mutie CWJPHN. Application of stable isotope dilution techniques to assess body fat and comparison with WHO BMl-for-age classification as a measure of obesity among schoolchildren in Nairobi, Kenya. 2020:1-5.

21. Tadesse BT, Foster BA, Kabeta A, Ayalew F, H/Meskel G, Jerene D, et al. Hepatic and renal toxicity and associated factors among HIV-infected children on antiretroviral therapy: a prospective cohort study. 2019;20(2):147-56. 
22. Beng H, Rakhmanina N, Moudgil A, Tuchman S, Ahn S-Y, Griffith C, et al. HIV-Associated CKDs in Children and Adolescents. 2020;5(12):2292-300.

23. Nguyen RN, Ton QC, Luong MH, Le LHLJHA. Long-Term Outcomes and Risk Factors for Mortality in a Cohort of HIV-Infected Children Receiving Antiretroviral Therapy in Vietnam. 2020;12:779.

24. Moy FS, Fahey P, Nik Yusoff NK, Razali KA, Nallusamy R, paediatrics TAPHODJJo, et al. Outcomes of human immunodeficiency virus-infected children after anti-retroviral therapy in M alaysia. 2015;51(2):204-8.

25. Teeraananchai S, Kerr SJ, Puthanakit T, Bunupuradah T, Ruxrungtham K, Chaivooth S, et al. Attrition and mortality of children receiving antiretroviral treatment through the universal coverage health program in Thailand. 2017;188:210-6. e1.

26. Zhu J, Yousuf MA, Yang W, Zhu Q, Shen Z, Lan G, et al. Mortality and Attrition Rates within the First Year of Antiretroviral Therapy Initiation among People Living with HIV in Guangxi, China: An Observational Cohort Study. 2021;2021.

27. UNICEF. Global and regional trends 2020 [5/13/2021]. Available from: https://data.unicef.org/topic/hivaids/global-regional-trends/.

28. Walker AS, Prendergast AJ, Mugyenyi P, Munderi P, Hakim J, Kekitiinwa A, et al. Mortality in the year following antiretroviral therapy initiation in HIV-infected adults and children in Uganda and Zimbabwe. 2012;55(12):1707-18.

29. Muri L, Gamell A, Ntamatungiro AJ, Glass TR, Luwanda LB, Battegay M, et al. Development of HIV drug resistance and therapeutic failure in children and adolescents in rural Tanzania: an emerging public health concern. 2017;31(1):61.

30. Mutanga JN, Mutembo S, Ezeamama AE, Song X, Fubisha RC, Mutesu-Kapembwa K, et al. Long-term survival outcomes of HIV infected children receiving antiretroviral therapy: an observational study from Zambia (2003-2015). 2019;19(1):1-12.

31. Shah M, Risher K, Berry SA, Dowdy DWJCid. The epidemiologic and economic impact of improving HIV testing, linkage, and retention in care in the United States. 2016;62(2):220-9. 